Gang Nam Lee

Kwang Hyo Jung

Young Jun Chae

Il Ryong Park

Sime Malenica

Yun Suk Chung

http://dx.doi.org/10.21278/brod67308

\title{
EXPERIMENTAL AND NUMERICAL STUDY OF THE BEHAVIOUR AND FLOW KINEMATICS OF THE FORMATION OF GREEN WATER ON A RECTANGULAR STRUCTURE
}

UDC 629.5.018.71:629.5.015.2:519.61

Original scientific paper

\begin{abstract}
Summary
In this study, the behaviour of green water impacting on a fixed rectangular structure is studied, and the flow kinematics is investigated with a series of experiments and computational fluid dynamic simulations. The experiments are conducted in a two-dimensional wave flume with the structure under regular wave conditions that are scaled down by the ratio of 1:125 from the BW Pioneer FPSO (Floating production storage and offloading) operated in the Gulf of Mexico. The mean values of the horizontal and vertical velocity profiles are provided for the water and bubbly flow induced by the interaction between the rectangular structure and regular waves. CFD simulations are also performed by STAR-CCM+ using the volume-of-fluid (VOF) method based on the finite-volume method (FVM) and all of CFD results are compared with the experimental data.
\end{abstract}

Key words: $\quad$ green water; rectangular structure; particle image velocimetry (PIV); bubble image velocimetry (BIV); computational fluid dynamics (CFD)

\section{Introduction}

When ships and offshore structures encounter a large wave in the ocean, the green water could occur on the deck, and it could cause severe damage to equipment and injuries to the members of the crew on the deck [1]. The bows of ships and offshore structures have been destroyed by the green water [2]. Many researchers have conducted investigations designed to understand the green water phenomena and to reduce the damage it causes. Experimental studies have been conducted to measure the distribution of water pressure on the deck and the superstructure of ships, and the shape of water shipping on deck has been investigated. Buchner and Voogt [3] investigated the water shipping pattern and the pressure distribution on the deck of FPSO due to green water with varying bow flare angles, which resulted in the faster water velocity under the water surface becoming lower on deck at the higher bow flare angle. 
Gang Nam Lee, Kwang Hyo Jung, Young Jun Chae, Il Ryong Park, Sime Malenica, Yun Suk Chung
Experimental and Numerical Study of the Behavior and Flow Kinematics of the Formation of Green Water on a Rectangular Structure

Greco [4] performed tests with a rectangular model in a 2-D wave tank and showed that the shape of the water overtopping the deck was similar to a plunging wave breaker. Hamoudi and Varyani [5] studied the probability of the occurrences of green water with varying wave heights and periods, vessel speeds, freeboard clearances, and bow shapes in the towing tank. Ryu and Chang [6] measured the water velocity fields of a plunging wave breaker at the front face of the deck and bubbly flow after overtopping of the deck using particle image velocimetry (PIV) and bubble image velocimetry (BIV) techniques. The velocity fields of bubbly flow on the deck were compared with the results of dam breaking solution. Varyani and Pham [7] applied a green water protector that had the shape of a whaleback forecastle to reduce the effect of green water effect on the deck of a high-speed container ship. They suggested what the appropriate slope of the whaleback forecastle-type green water protector should be to decrease the green water loading.

Several numerical studies have been conducted to examine the green water effect, and various numerical methods have been used to simulate its complicated phenomena. Nielsen and Mayer [8] solved the Navier-Stokes equation using the volume of fluid (VOF) method to capture the shape of the free surface, and the results were compared with the experimental results in Greco's 2-D wave tank [4] and with Buchner and Voogt's towing-tank [3] tests of the effect of green water on FPSO. Yamasaki et al. [9] applied the finite difference method and used the density function method and overlapping grid systems to simulate the green water phenomena and the hydrodynamic motion of a rectangular model. Their numerical results were in good agreement with their own pressure measurements. Shibata and Koshizuka [10] used the numerical particle method for the green water phenomena on a fixed deck and compared their experimental results to those obtained by Tanizawa et al. [11] in 2-D wave tank.

Most of the recent research related to the green water phenomena has been focused on the characteristics of the flow, analyses pressure distributions, and impact loading on various deck and superstructures using both experimental and numerical methods. However, it is also important to analyse the behaviour of green water and the kinematics of flow at the weatherside and on the deck of ships and offshore structures. Investigation of the wave deformation and kinematics of water flow and bubbly flow due to the green water on a rectangular structure would be amenable to simulations using computational fluid dynamics.

In this study, the green water phenomena on the rectangular structure fixed in 2-D wave tank are investigated with two velocity measurement techniques, i.e., PIV for the water velocity profiles and BIV for bubbly flow. The behaviour of the formation of green water on the rectangular structure is categorized into five different steps, i.e., flip through, air entrapment, wave run-up, wave overturning, and water shipping. The velocity profiles in each phase are provided at the weather side and on the deck of the rectangular structure in water and in the bubbly flow region. Computational fluid dynamic simulation using STAR-CCM+ is used with the finite volume method to solve the Navier-Stokes equation, and the VOF method is used to determine the free surface. These calculations are performed for all of the experimental conditions, and the results are compared with the experimental results of the wave transforming shape and water kinematics induced by green water on the rectangular structure in 2-D wave tank. 
Experimental and Numerical Study of the Behavior and Flow Kinematics of the Formation of Green Water on a Rectangular Structure
Gang Nam Lee, Kwang Hyo Jung Young Jun Chae, Il Ryong Park, Sime Malenica, Yun Suk Chung

\section{Experimental setup and technique}

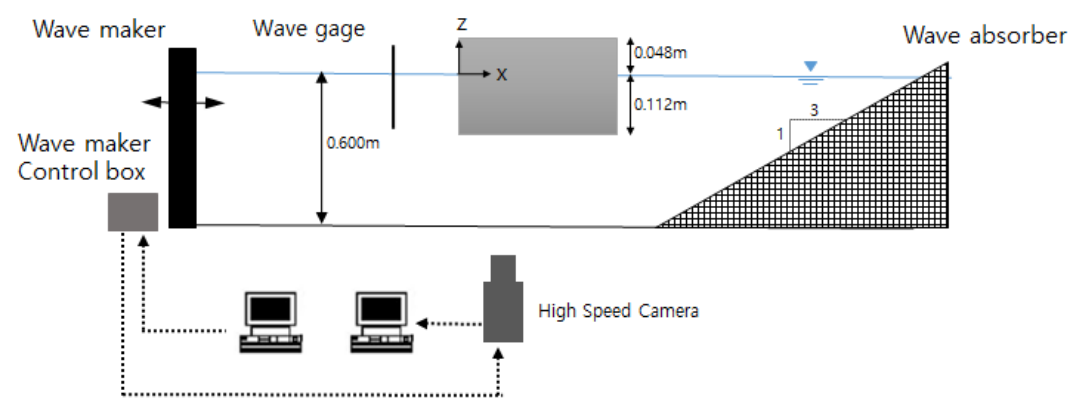

Fig. 1 Schematic view of the experimental setup

A series of experiments to investigate the green water phenomena are carried out in the 2-D wave tank, which was $32 \mathrm{~m}$ long, $0.6 \mathrm{~m}$ wide, and $1 \mathrm{~m}$ deep. The 2-D wave tank is equipped with a piston-type wave maker, and it has a sloped beach (1:3) to absorb waves at the end of the tank. The acrylic, rectangular-shaped model is built with a scale ratio of 1:125 based on the FPSO BW Pioneer that has been operating in the Gulf of Mexico since 2008. The rectangular structure is fixed with an alloy frame at the location of $15 \mathrm{~m}$ from the wave maker. The wave characteristics are chosen in order to model the conditions of the Gulf of Mexico. In that respect the regular waves with the period of $1.16 \mathrm{~s}(13 \mathrm{~s}$ at full scale) are generated for three different wave heights. The significant wave height $\left(H_{1 / 3}\right)$ of $12.5 \mathrm{~m}$ for the 100 -year return period is referred in the west central Gulf of Mexico [12], where FPSO BW Pioneer has operated. In addition two regular waves $\left(\mathrm{H}_{1 / 10}\right.$ and $\left.\mathrm{H}_{1 / 100}\right)$ that are steeper than $H_{1 / 3}$ are determined using the Equation (1) [13].

$$
H_{1 / n}=n \sqrt{2 m_{0}}\left[\frac{1}{n} \sqrt{\log _{e} n}+\sqrt{\pi}\left\{1-\operatorname{erf}\left(\sqrt{\left.\log _{e} n\right\}}\right]\right.\right.
$$

Where $H_{1 / n}$ is the average of the high $\mathrm{n}^{\text {th }}$ of waves and $m_{0}$ is the $0^{\text {th }}$ moment of the wave energy spectrum. The water depth is kept at $0.6 \mathrm{~m}$ for all of the experiments. Detailed information of the experimental model and the regular waves are provided in Tables 1 and 2. Because the experiment is performed in 2-D wave tank, the model is fixed at the condition of wall to wall in the wave tank having its width of $0.60 \mathrm{~m}$, and the length of model is not followed by the scale ratio.

Table 1 Principle dimension of 1/125 model of FPSO

\begin{tabular}{|c|c|c|}
\hline & BW Pioneer & Model \\
\hline Length (m) & 241 & N/A \\
\hline Breadth (m) & 42 & 0.336 \\
\hline Depth (m) & 20.4 & 0.163 \\
\hline Draft (m) & 13.9 & 0.111 \\
\hline Freeboard (m) & 6.5 & 0.052 \\
\hline
\end{tabular}

Table 2 Wave conditions of model test in a 2-D wave tank

\begin{tabular}{|c|c|c|}
\hline & West central of GOM & Model test \\
\hline \multirow{3}{*}{ Wave height $(\mathrm{m})$} & & $0.100\left(H_{1 / 3}\right)$ \\
& $12.5\left(H_{1 / 3}\right)$ & $0.127\left(H_{1 / 10}\right)$ \\
& & $0.166\left(H_{1 / 100}\right)$ \\
\hline Peak period $(\mathrm{s})$ & 13 & 1.16 \\
\hline
\end{tabular}


Gang Nam Lee, Kwang Hyo Jung, Young Jun Chae, Il Ryong Park, Sime Malenica, Yun Suk Chung
Experimental and Numerical Study of the Behavior and Flow Kinematics of the Formation of Green Water on a Rectangular Structure

The particle image velocimetry (PIV) technique is used to obtain the velocity field under the free surface on the weather side of the structure, with the exception of the bubbly flow region.

The PIV system and the wave maker are synchronized by a computer, and PIV images are obtained at the acquisition rate of $500 \mathrm{~Hz}$ with the digital CCD camera (Redlake Y5) mounted with a $105-\mathrm{mm}, \mathrm{f} / 1.8$ macro focal lens set at $\mathrm{f} / 2.8$. The two sizes of the field of view (FOV) are $147 \mathrm{~mm}$ and $200 \mathrm{~mm}$, providing a FOV area of $147 \times 200 \mathrm{~mm}^{2}$ at the weather side of the rectangular structure. The spatial resolution is $2.72 \mathrm{~mm}$ between all of the velocity vectors in the interrogation area of $64 \times 64$ pixels with $50 \%$ overlap (Figure 2(a)). The continuous laser (Maximum 8 watts, $532 \mathrm{~nm}$ ) is used to illuminate the particles in the water. The PIV particles have mean diameter of $57 \mu \mathrm{m}$ and a specific gravity of 1.02. The adaptive multi-pass algorithm is used to get a stronger cross-correlation to reduce faulty vectors, and spurious false vectors are eliminated by the median filter [14].

The bubble image velocimetry (BIV) technique is applied to measure the velocity profiles in the bubbly flow at the front of the rectangular structure and on its deck. BIV images are obtained by the CCD camera's $50 \mathrm{~mm} \mathrm{f} / 1.8$ macro focal lens set at $\mathrm{f} / 4.0$ and using the shadowgraph technique at the image acquisition rate of $500 \mathrm{~Hz}$; the size of the FOV was $360 \times$ $264 \mathrm{~mm}^{2}$. Spatial resolutions are $2.44 \mathrm{~mm}$ between the vectors in the interrogation area of 32 $\times 32$ pixels with $50 \%$ overlap (Figure 2(b)). The velocity vectors of the BIV images are calculated by the minimum quadratic difference (MQD) method [15].

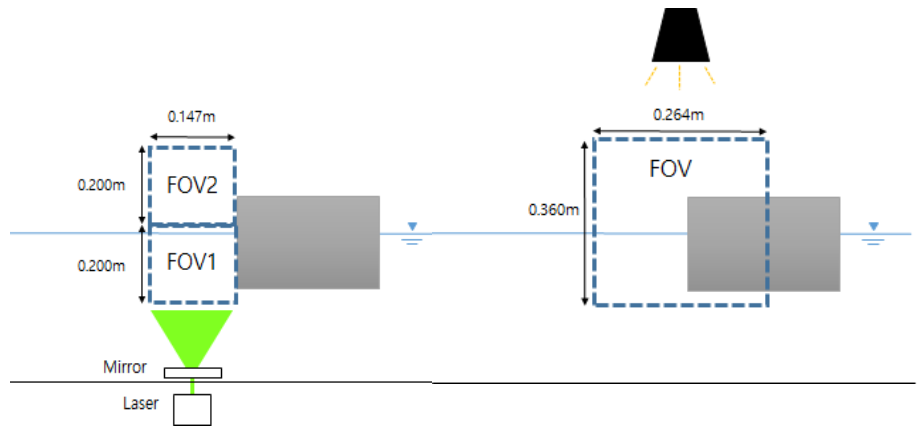

(a) FOVs of PIV

(b) FOV of BIV

Fig. 2 Field of View for PIV and BIV measurement

\section{Computational setup}

Computational fluid dynamic (CFD) analysis using STAR-CCM+ is used to simulate the green water phenomena on the rectangular structure based on the finite volume method. The governing equations for incompressible, two-phase flows, the continuity equation (Equation (2)), and the Navier-Stokes equation (Equation (3)) are solved with the assumptions of isothermal conditions and laminar flow.

$$
\begin{gathered}
\frac{\partial u_{i}}{\partial x_{i}}=0 \\
\frac{\partial u_{i}}{\partial t}+u_{i} \frac{\partial u_{i}}{\partial x_{j}}=-\frac{1}{\rho} \frac{\partial p}{\partial x_{i}}+v \frac{\partial^{2} u_{i}}{\partial x_{j} \partial x_{j}}+g_{i}
\end{gathered}
$$

Where $u_{i}$ is fluid velocity, $\rho$ is fluid density, $t$ is time, $x_{i}$ is the coordinate, $p$ is pressure, $v$ is the coefficient of the kinematic viscosity of the fluid, and $g_{i}$ is gravitational acceleration. 
Experimental and Numerical Study of the Behavior and Flow Kinematics of the Formation of Green Water on a Rectangular Structure
Gang Nam Lee, Kwang Hyo Jung Young Jun Chae, Il Ryong Park, Sime Malenica, Yun Suk Chung

To solve the governing equations, the second-order numerical schemes are used for both time and spatial discretization, and the semi-implicit method for pressure-linked equation (SIMPLE) method is used to couple the velocity and pressure.

The volume of fluid (VOF) method (Equation (4) [16]) is used to capture the interface between the air and the water.

$$
\frac{\partial}{\partial t} \int_{V} \alpha d V+\int_{S} \alpha \vec{v} \cdot \vec{n} d S=0
$$

where $\alpha$ is the volume ratio that determines the state in the grid. $V$ and $S$ present the control volume and the control surface, respectively. The value of $\alpha$ varies from 0 in the air region to 1 in the water region, and the value is in the range of $0<\alpha<1$ at the interface between the air and the water.

The computational domain is $10 \mathrm{~m}$ long, $0.6 \mathrm{~m}$ wide, and $1.6 \mathrm{~m}$ high, and the rectangular structure is located at $4 \mathrm{~m}$, as shown in Figure 3. The waves are introduced into the domain at the inlet boundary through the inlet velocity boundary condition and they are extracted from the domain at the outlet boundary through the pressure outlet boundary condition. Symmetry condition is applied at the boundaries at the top and side walls of the computational domain, and no-slip wall condition is used at the bottom of the computational domain and the surface of the rectangular structure. The numerical wave damping method [17] is used at the end of the computational domain to dissipate the energy induced by the wave and to reduce the reflection of the wave. Trimmer mesh is generated in all of the computational domains to ensure that the structure of the mesh is high quality and has good anisotropic refinement [18]. The computational setup is decided from a series of simulations based on the convergence of the incoming wave height with varying mesh sizes and time steps, which are one hundredth of the wave length for the number of mesh in horizontal direction, one fiftieth of each wave height for vertical direction near the free surface, and one thousandth of the wave period for the computational time step. The horizontal velocity profiles of the water particles are simulated by CFD and verified by comparing them to the Stokes' third-order theory and PIV experimental results. Figure 4 shows that there was good agreement with the averaged discrepancies having $3.7 \%$ for experimental results and $6.71 \%$ for CFD results against the results of Stokes' third order theory. To simulate bubbly flows due to the interaction of waves and the structure, the finer sized meshes $(1.5 \mathrm{~mm})$ were used near the structure, because the VOF method requires at least three cells across each bubble to produce less modelling errors [18]. The total number of cells in the computational domain is around six million (Figure 5), and the CPU time required to compute five consecutive wave periods of regular waves is about 39 hours using two Intel Xeon E5-2697, 2.60-GHz processors.

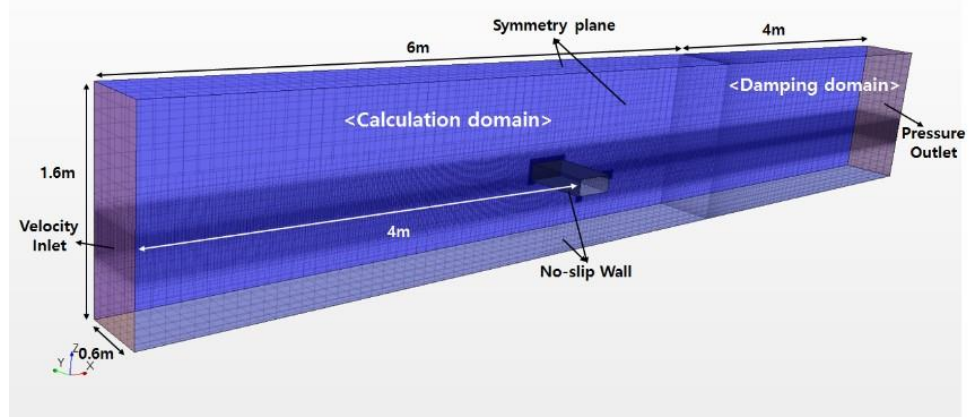

Fig. 3 Computational domain and boundary conditions of CFD simulation 
Gang Nam Lee, Kwang Hyo Jung, Young Jun Chae, Il Ryong Park, Sime Malenica, Yun Suk Chung
Experimental and Numerical Study of the Behavior and Flow Kinematics of the Formation of Green Water on a Rectangular Structure

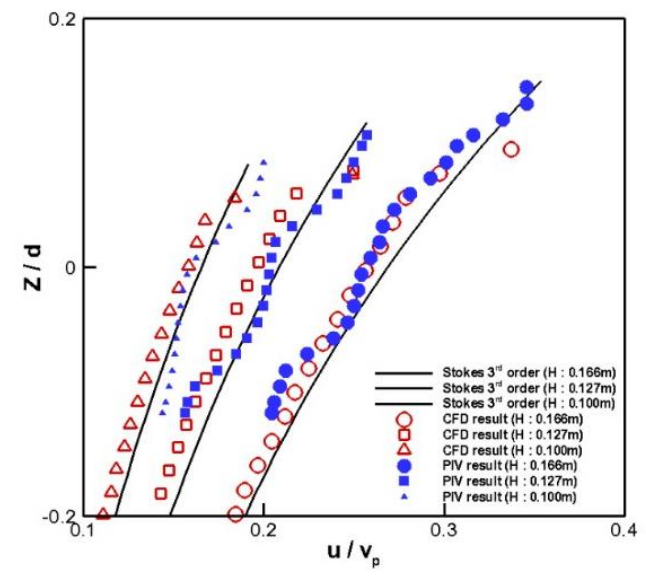

Fig. 4 Comparison of the horizontal velocity profiles of water particles

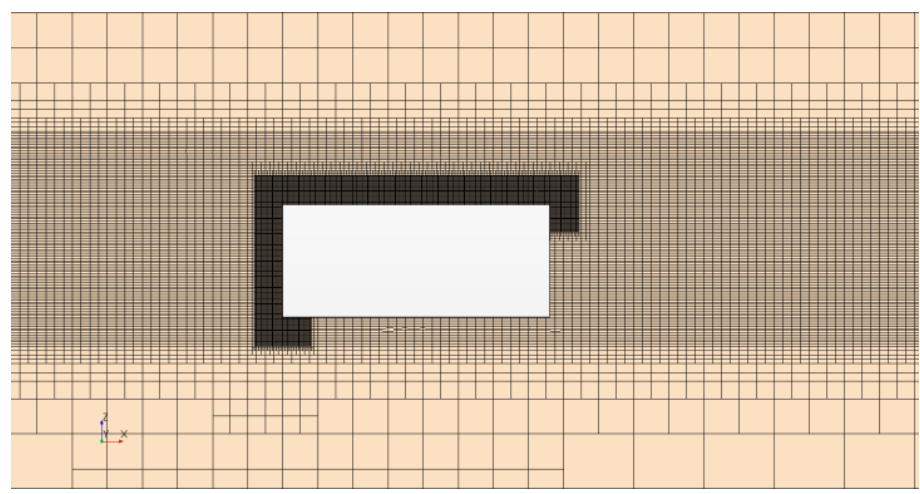

Fig. 5 Mesh setup for CFD analysis

\section{Experimental and simulation results}

\subsection{Behaviour of green water formation}

When a large wave encounters a structure in the ocean environment, it is deformed, reflected, transmitted, and overtopped due to the interaction between the wave and the structure, which causes the green water phenomena. In this study, the green water phenomenon on the rectangular structure due to regular waves is investigated with experiments using optical techniques (PIV and BIV) and CFD simulations. The process by which the green water develops is referred to as the behaviour of green water formation and it consists of five distinct steps. Instantaneous images are taken by the CCD camera during the generation of green water over the rectangular structure in the 2-D wave tank for the wave condition of $\mathrm{H}_{1 / 100}$, as shown in Figure 6(a). The first step in Figure 6(a) shows that the shape of the wave is deformed vertically with a concave face before the wave front impacted on the weather side of the structure. It is mentioned for the similar phenomena of a wave impacting on a vertical wall and named the flip-through phase $[19,20]$. The second step is air being entrapped on the weather side of the structure, as shown in Figure 6(b), which can be named the air entrapment phases [20]. Figure 6(c) shows that the third step occurred when the water level increases along the weather side and splashes up into air; this is called the wave run-up phase [21]. The wave that was splashed up into the air overturns like the plunging wave breaker and impacts on the deck, as shown in Figure 6(d). This occurrence is called the wave overturning phase [22]. After the overturned wave impinges on the deck, the body of bubbly water is moved forward along the deck, as shown in Figure 6(e), and this is called the water shipping phase [4]. 


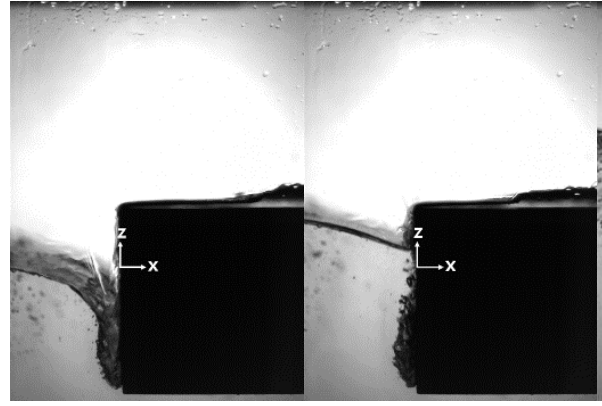

(a)

(b)

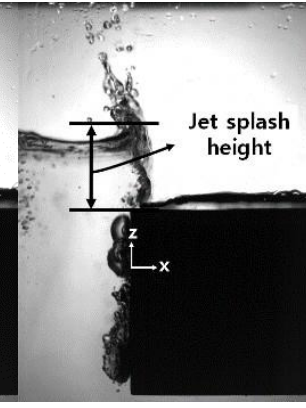

(c)

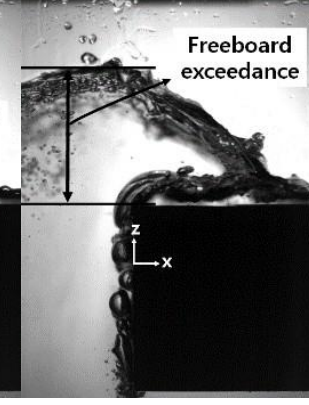

(d)

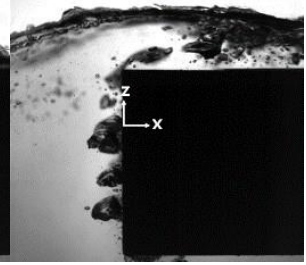

(e)

Fig. 6 Snapshots of the generation of green water in the 2-D experiment in wave condition of $H_{1 / 100}$

In summary, the generation of green water on the rectangular structure consists of five phases, i.e., the flip-through, air-entrapment, wave run-up, wave overturning, and water shipping phases.

CFD simulation is performed for the green water on the rectangular structure at the same experimental conditions, and similar results are obtained for the key phenomenon at each phase. Figure 7 shows that these phenomena consist of the shape of the free surface at the flip-through phase, the size and location of the aerated region at the air entrapment phase, the height of jet splash at the wave run-up phase, the water overturning shape and impinging location at the wave overturning phase, and the level of the water at the water shipping phase. But there are some differences in behaviours of bubble and water droplet during developing the green water. For instance, at the wave run-up phase, the water droplets splashed up in the air was found in the experiment, but it is not simulated with CFD technique. The bubbly flow simulated by CFD technique has the qualitatively similar pattern with the experimental measurement, but it shows the some discrepancy of the size and detail behaviour of bubbles near the structure in comparison of CFD simulation results (Figure 7) and images taken from experiment (Figure 6).

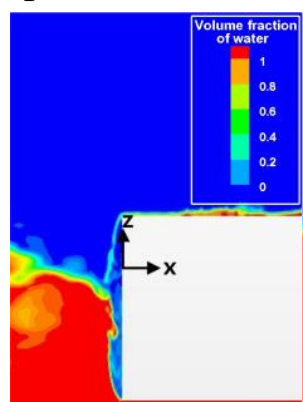

(a)

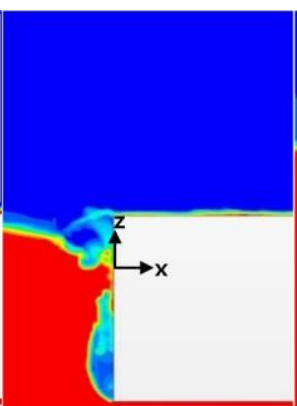

(b)

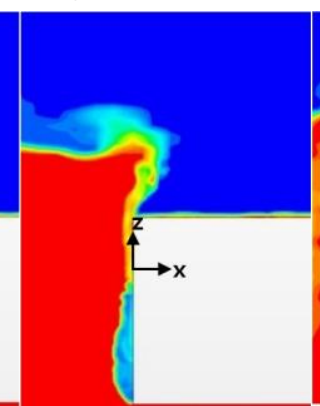

(c)

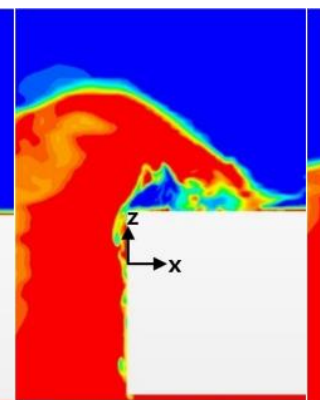

(d)

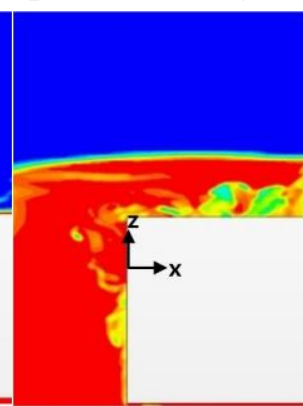

(e)

Fig. 7 Volume of fluid (VOF) scene of CFD simulation in wave condition of $H_{1 / 100}$

The height of the jet splash $\left(H_{J}\right)$ is defined as the maximum height of the water at the wave run-up phase from the deck and the freeboard exceedance $\left(H_{F}\right)$ to be the highest water level at the wave overturning phase from the deck, as shown in Figure 8. The jet splash height and the freeboard exceedance induced by the green water increase linearly with the height of incoming regular wave. The results of the experiments and CFD simulations are compared in Figure 8 and agree well with the averaged difference of $5.6 \%$ between them. 
Gang Nam Lee, Kwang Hyo Jung, Young Jun Chae, Il Ryong Park, Sime Malenica, Yun Suk Chung
Experimental and Numerical Study of the Behavior and Flow Kinematics of the Formation of Green Water on a Rectangular Structure

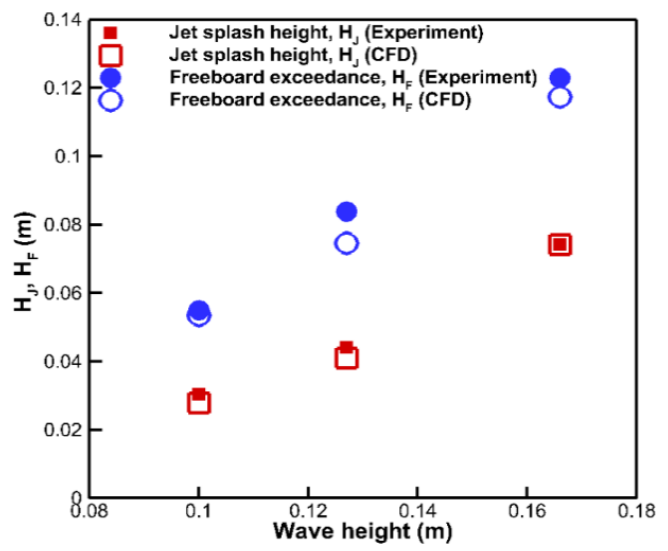

Fig. 8 Comparison of the height of jet splash and freeboard exceedance with CFD results at each wave condition

\subsection{Flow kinematics of green water}

PIV and BIV techniques are applied to measure the velocity fields for water and bubbly flow regions near the rectangular structure. To investigate the kinematics of the water and the bubbly flow near the structure, the flow fields are assigned to three analysis regions, i.e., 1) water flow at the weather side (WFW, $\left.20 \times 360 \mathrm{~mm}^{2}\right)$, 2) bubbly flow at the weather side (BFW, $20 \times 360 \mathrm{~mm}^{2}$ ), and 3) bubbly flow on the deck (BFD, $160 \times 40 \mathrm{~mm}^{2}$ ), as shown in Figure 9 . After obtaining the velocity profiles from two FOVs of PIV as shown in Figure 2, spatial mean velocities are obtained every $10 \mathrm{~mm}$ in the vertical direction within the WFW region. In the bubbly flow region, spatial mean velocities are averaged for $20 \times 10 \mathrm{~mm}^{2}$ in the BFW region, and they are averaged for $10 \times 40 \mathrm{~mm}^{2}$ in the BFD region from the velocity profiles measured by BIV. The measured spatial mean velocity distributions in the three analysis regions for the five phases of the green water formation is compared to CFD results in Figures 10 to 15 . The $x$ and $y$ axes are non-dimensionalized with the breadth of structure $(B=0.336 \mathrm{~m})$ and the depth of the water $(d=0.6 \mathrm{~m})$, respectively.

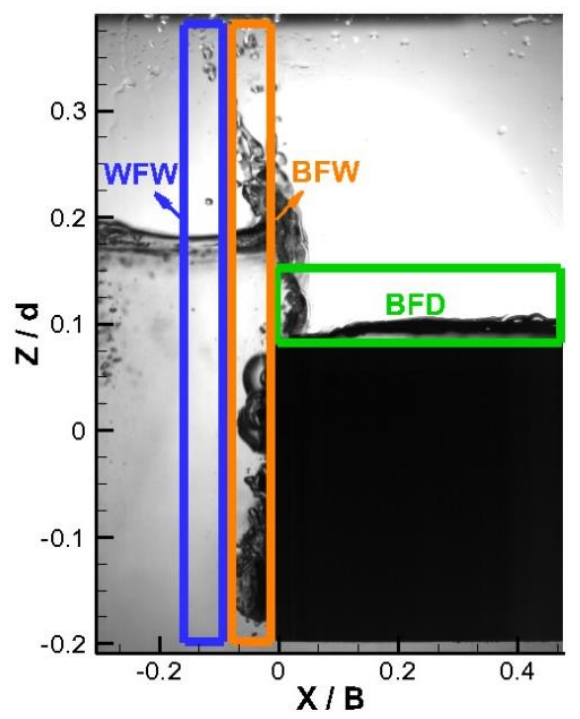

Fig. 9 Analysis region for computing velocity profiles for PIV and BIV measurements: Analysis region of water flow at weather side (WFW), bubbly flow at weather side (BFW), bubbly flow at deck side (BFD).

Horizontal and vertical mean velocities in the WFW region during the flip-through phase are shown for each wave condition in Figure 10. Velocities are non-dimensionalized with the phase velocity, $v_{p}=1.72 \mathrm{~m} / \mathrm{s}$ of the regular wave $(T=1.16 \mathrm{~s})$ at $d=0.6 \mathrm{~m}$. At this phase, the water has the negative horizontal velocity due to the return flow under the bottom. It curls in 
Experimental and Numerical Study of the Behavior and Flow Kinematics of the Formation of Green Water on a Rectangular Structure
Gang Nam Lee, Kwang Hyo Jung Young Jun Chae, Il Ryong Park, Sime Malenica, Yun Suk Chung

the clockwise direction to the weather side of the structure and changes to the positive horizontal direction near the free surface. Then, the wave front deforms, forming a concave face. The water keeps moving upward with the free surface rising and has its maximum vertical velocity at the level of the zero horizontal mean velocity. There is a good agreement between results of CFD simulation and experiments in differences of $0.0002 v_{p}$ for $\mathrm{H}_{1 / 3}, 0.06 v_{p}$ for $\mathrm{H}_{1 / 10}$, $0.01 v_{p}$ for $\mathrm{H}_{1 / 100}$ for horizontal direction and $0.04 v_{p}$ for $\mathrm{H}_{1 / 3}, 0.02 v_{p}$ for $\mathrm{H}_{1 / 10}, 0.02 v_{p}$ for $\mathrm{H}_{1 / 100}$ for vertical direction..

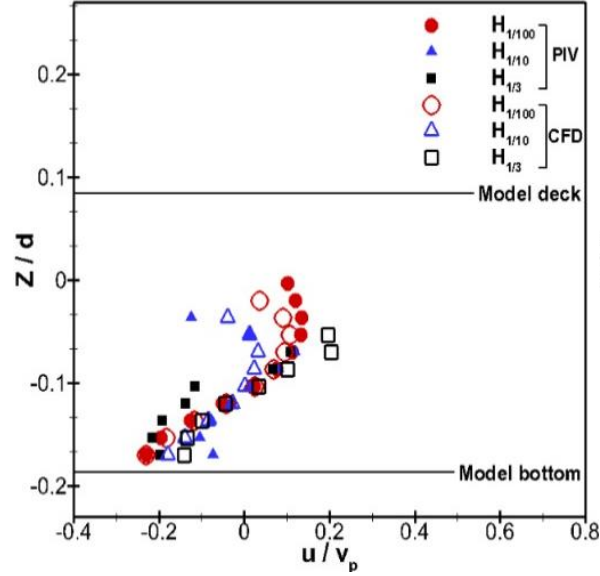

(a) Horizontal velocity

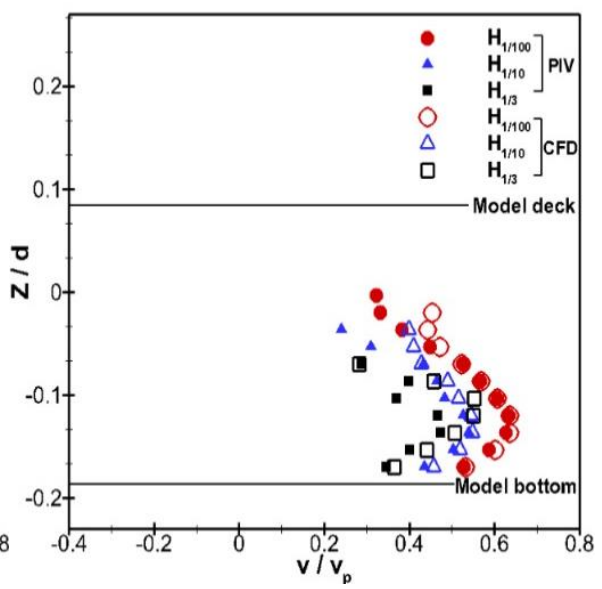

(b) Vertical velocity

Fig. 10 Comparison of velocity distributions obtained from PIV experiments and CFD simulations at the weather side in the flip-through phase

When the wave front hit the structure's wall, the bubbles are trapped on the weather side. This phase is called the air entrapment phase. Figure 11 shows the mean velocities of bubbly flow that are measured by the BIV technique. The measurements of the horizontal and vertical mean velocities of bubbly flow were nearly zero, because the bubbles are stagnated on the wall of the structure. However, the vertical mean velocity of the CFD simulation is relatively larger close to the free surface. This difference might be caused by the large bubbles, which are trapped on the wall, breaking down into the smaller bubbles, which move rapidly and are simulated in CFD but are not accounted for by BIV technique.

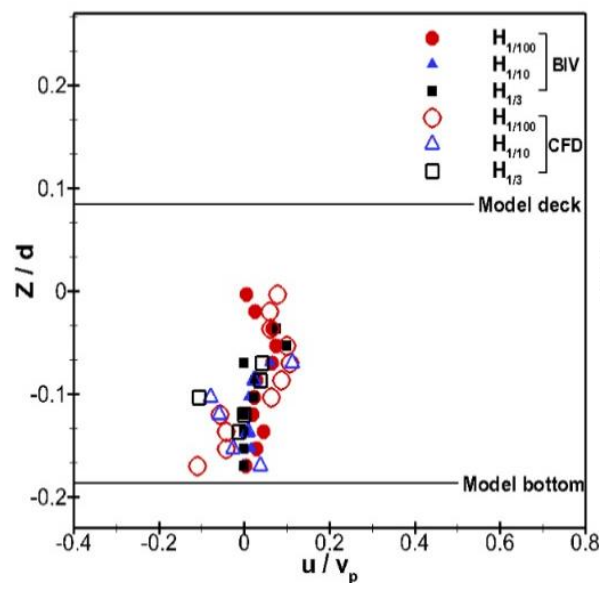

(a) Horizontal velocity

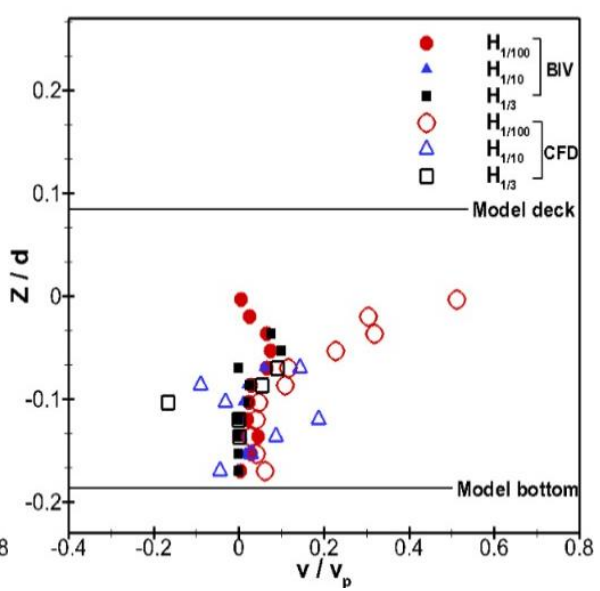

(b) Vertical velocity

Fig. 11 Comparison of velocity distributions obtained from BIV experiments and CFD simulations at the weather side in the air-entrapment phase

At the wave run-up phase, the water and bubbly flow have risen along the wall of the structure, and the water splashes over the deck level. A part of the bubbly flow separates from 
Gang Nam Lee, Kwang Hyo Jung, Young Jun Chae, Il Ryong Park, Sime Malenica, Yun Suk Chung
Experimental and Numerical Study of the Behavior and Flow Kinematics of the Formation of Green Water on a Rectangular Structure

the entrapped bubble and moved upward along the wall, as shown in Figure 6(c), and it has the positive mean vertical velocity of only 0.4 times of the velocity of the wave phase in Figure 12. The lower part of the bubbly flow still is stagnated, and its horizontal and vertical mean velocities are close to zero, as shown in Figure 12. From the CFD results, the vertical mean velocities of the bubbly flow are relatively larger than the BIV measurement. This occurs for the same reason as in the air entrapment phase, in which BIV images can not capture the smallsized bubbles. Because bubbles is not found close to the deck at the weather side of structure from CFD simulation as shown in Figure 7(c) but bubbles are moved up to the deck along the structure in the model test (Figure 6(c)), it shows the discrepancy in the level of velocity distribution in Figure 12.

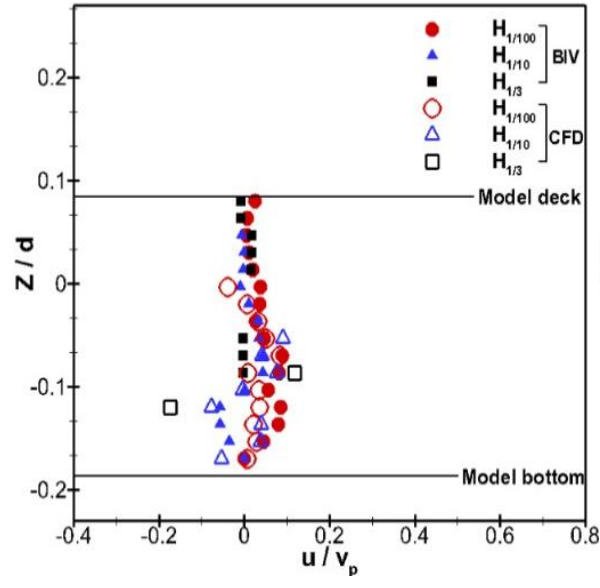

(a) Horizontal velocity

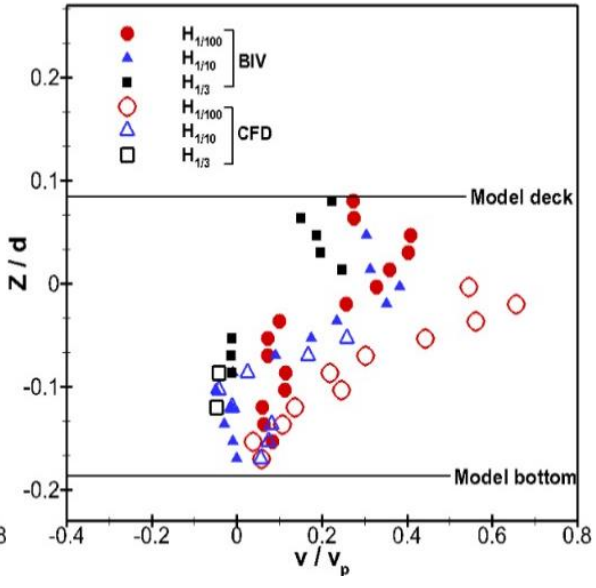

(b) Vertical velocity

Fig. 12 Comparison of velocity distributions obtained from BIV experiments and CFD simulations at the weather side in the wave run-up phase

When the wave is overturned and impacted on the deck, the shape of the wave is similar to a plunging wave breaking. The point at which the wave impinges on the deck moved further from the structure's upper corner when the height of the incoming wave increases. Figure 13 shows that the horizontal mean velocities fluctuated along the deck after the wave overturned at the corner, and they are faster at the point of impingement. The horizontal mean velocities of the bubbly flow on the deck are affected by the overturning wave flowing over the corner of the structure. They are also affected by the collapse of the bubbles, which include complicated vortical flow. The vertical mean velocities decrease gradually, reaching their lowest value at the point of impingement. The horizontal mean velocities of the CFD results are underestimated in comparison to the BIV measurements before the point of impingement, because they are averaged spatially as the bubbly flow rotated in the clockwise direction on the deck under the overturning wave. This rotating bubbly flow is not observed in the experiments due to the lack of spatial resolution of the BIV measurements. However, the rotated and collapsed bubbly flow is observed visually during the wave overturning phase. 
Experimental and Numerical Study of the Behavior and Flow Kinematics of the Formation of Green Water on a Rectangular Structure
Gang Nam Lee, Kwang Hyo Jung Young Jun Chae, Il Ryong Park, Sime Malenica, Yun Suk Chung

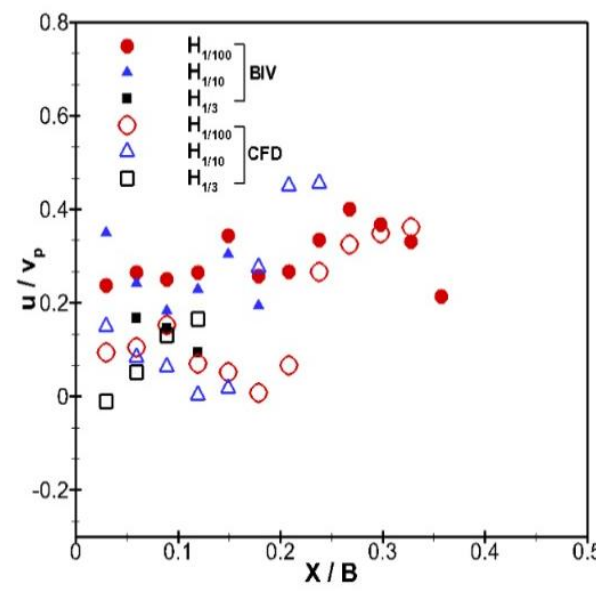

(a) Horizontal velocity

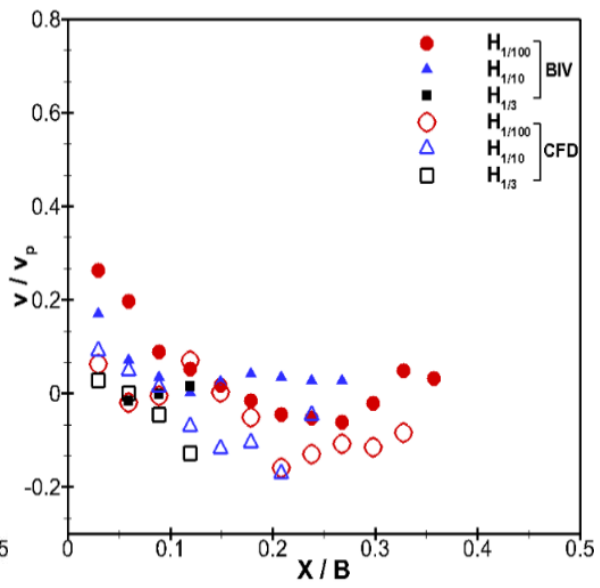

(b) Vertical velocity

Fig. 13 Comparison of velocity distributions obtained from BIV experiments and CFD simulations at the deck side in the wave overturning phase

After the wave overturned and fell onto the deck, the water surges along the deck, and this is called the water shipping phase. The horizontal mean velocities of bubbly flow increase gradually up to $0.4 v_{p}$ at the location of $0.3 \mathrm{~B}$ under the conditions of $H_{1 / 100}$ and $H_{1 / 10}$ and up to $0.27 v_{p}$ at the location of $0.2 \mathrm{~B}$ for $H_{1 / 3}$. Later these locations fluctuate with the bubble movement. In the water shipping phase, the surging flow in the horizontal direction is mostly dominant, but the vertical flow is confined under the free surface, and it is close to zero on the deck, as shown in Figure 14(b). The spatial mean velocities of CFD has reasonably a good agreement with the BIV measurements in differences of $0.11 v_{p}$ for $\mathrm{H}_{1 / 3}, 0.09 v_{p}$ for $\mathrm{H}_{1 / 10}, 0.08$ $v_{p}$ for $\mathrm{H}_{1 / 100}$ for horizontal direction and $0.04 v_{p}$ for $\mathrm{H}_{1 / 3}, 0.07 v_{p}$ for $\mathrm{H}_{1 / 10}, 0.02 v_{p}$ for $\mathrm{H}_{1 / 100}$ for vertical direction. Those quantitative discrepancies are caused by differences in the bubble behaviours and distribution between CFD simulations and experiments.

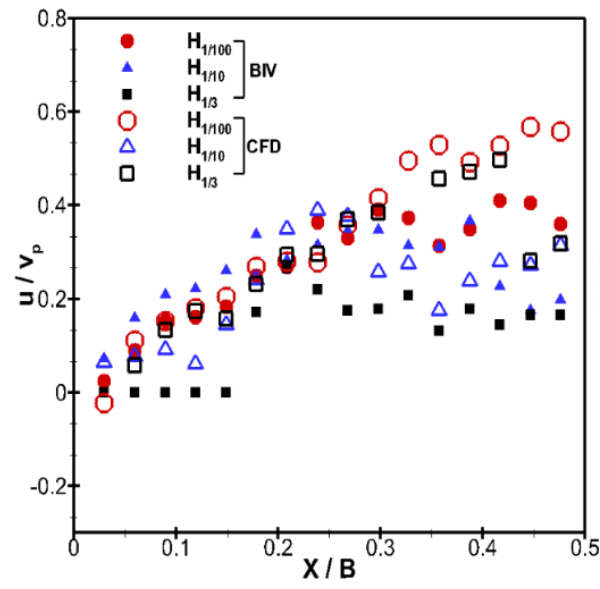

(a) Horizontal velocity

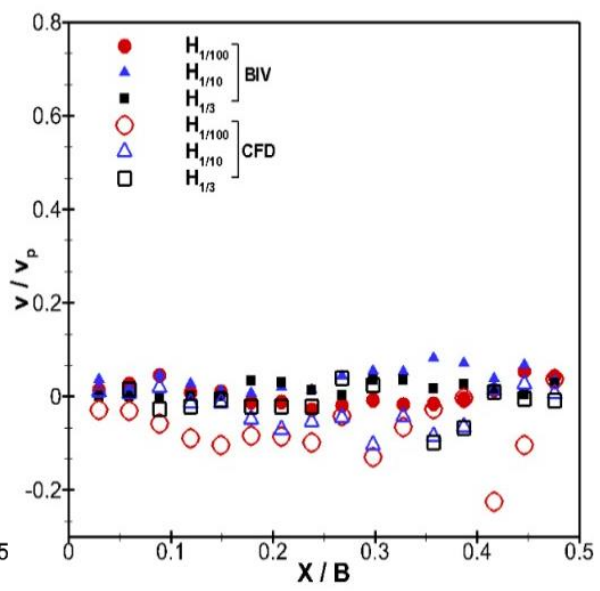

(b) Vertical velocity

Fig. 14 Comparison of velocity distributions obtained from BIV experiments and CFD simulations at the deck side in the water shipping phase

\section{Conclusions}

Green water phenomena on the fixed rectangular structure is investigated with experiments performed in the 2-D wave tank and by CFD simulation using STAR-CCM+. PIV 
Gang Nam Lee, Kwang Hyo Jung, Young Jun Chae, Il Ryong Park, Sime Malenica, Yun Suk Chung
Experimental and Numerical Study of the Behavior and Flow Kinematics of the Formation of Green Water on a Rectangular Structure

and BIV measurements are applied to obtain the water and bubbly flow kinematics induced by the green water.

The process by which green water is generated was categorized into five phases, i.e., flip through, air entrapment, wave run-up, wave overturning, and water shipping, which aid in understanding the generation of green water on ships and offshore structures. The noticeable water levels during the generation of the green water, which are the height of the jet splash $\left(H_{J}\right)$ and the freeboard exceedance $\left(H_{F}\right)$, are defined, and their relationship with the heights of the incoming waves are presented.

The spatial distributions of mean velocity at the weather side and over the deck of the rectangular structure are provided at the water and bubbly flow regions for the five phases categorized in this study. The complicated bubbly flow is developed at the weather side and over the deck, and the bubbles' behaviours have collapsing and rotating movements along the surface of the structure.

The green water phenomena are simulated with CFD in 3-D using the finite volume method and the volume of fluid model. The computational setup, including the grid size, the time step, and the boundary conditions, is provided to simulate the green water phenomena on the rectangular structure.

The CFD simulation results into the free surface shape being deformed at each phase of green water formation to be similar to the experimental measurements. The height of the jet splash $\left(H_{J}\right)$ and the freeboard exceedance $\left(H_{F}\right)$ are simulated in CFD, and there is good agreement with the experimental results. Also, the distributions of the spatial mean velocities of the water and bubbly flow induced by the green water are analysed using the CFD results, and the results are compared with experimental results at each phase of green water generation. The distributions of spatial mean velocity show patterns that were similar quantitatively for the CFD results and the experimental results in the water flow region and in the bubbly flow region, in which the bubbles' behaviours are sustained in size and irrotational. When the bubbles are broken into smaller bubbles and rotated, the spatial mean velocity of the bubbly flows resulting from CFD had quantitative differences with the BIV measurements. This might be caused by the discrepancy of spatial resolution in the BIV measurements and the CFD simulation. To investigate the bubbly flow due to the green water with a better accuracy, higher spatial resolution for the BIV measurements would be required to obtain the detailed behaviours of the bubbles and to obtain a finer grid system and time step for the CFD simulation.

In summary we can say that, for this particular case of the fixed floating body with vertical walls, the numerical CFD simulations can predict the green water behaviour with good accuracy. The future investigations which are planned will include the floating body free to move and the walls with different inclination angles. The measurements of the local pressures at different locations will also be included in order to properly evaluate the hydrodynamic loading which is required for the structural analysis of the deck and superstructures. In addition, it is worth of the CFD computation to be performed at the compressible flow condition to understand the influence of compressibility on the bubble behaviour and the water kinematics near the structure in comparison of the present CFD results under the incompressible flow condition.

\section{ACKNOWLEDGEMENTS}

This work was supported by the National Research Foundation of Korea (NRF) grant funded by the Korea government (MSIP) through GCRC-SOP(No. 2011-0030013) . 
Experimental and Numerical Study of the Behavior and Flow Kinematics of the Formation of Green Water on a Rectangular Structure
Gang Nam Lee, Kwang Hyo Jung Young Jun Chae, Il Ryong Park, Sime Malenica, Yun Suk Chung

\section{REFERENCES}

[1] Faulkner D. 2001. Survival Design of Cargo Hatch Structures. RINA Conference Design and Operation for Abnomal Conditions II, London, United Kingdom.

[2] Ersdal G, Kvitrud A. Green water on Norwegian production ships. Proceedings of the The Tenth Int. Offshore and Polar Engineering Conference; 2000: Int. Society of Offshore and Polar Engineers.

[3] Buchner B, Voogt A. 2000. The effect of bow flare angle on FPSO green water loading. OMAE2000.

[4] Greco M. 2001. A two-dimensional study of green-water loading. PhD Thesis. University of Trondheim.

[5] Hamoudi B, Varyani K. 1998. Significant load and green water on deck of offshore units/vessels. Ocean Engineering.25:715-731. http://dx.doi.org/10.1016/S0029-8018(97)10005-1.

[6] Ryu Y, Chang K-A. Breaking wave impinging and green water on a two-dimensional offshore structure. Proceedings of the The Fifteenth International Offshore and Polar Engineering Conference; 2005: International Society of Offshore and Polar Engineers.

[7] Varyani K, Pham X. 2008. Whaleback forecastle for reducing green water loading on high-speed container vessels. Ships and Offshore Structures.3:229-237. .http://dx.doi.org/10.1080/17445300802057407.

[8] Nielsen KB, Mayer S. 2004. Numerical prediction of green water incidents. Ocean Eng..31:363-399. http://dx.doi.org/10.1016/j.oceaneng.2003.06.001.

[9] Yamasaki J, Miyata H, Kanai A. 2005. Finite-difference simulation of green water impact on fixed and moving bodies. J.of marine science \& technology.10:1-10.http://dx.doi.org/10.1007/s00773-005-0194-1.

[10] Shibata K, Koshizuka S. 2007. Numerical analysis of shipping water impact on a deck using a particle method. Ocean Engineering.34:585-593. http://dx.doi.org/10.1016/j.oceaneng.2005.12.012.

[11] Tanizawa K, Sawada H, Hoshino K, Tujimoto M, Koshizuka S. Experimental and numerical study of shipping water impact on running ship foredeck in regular head seas. Proceedings of the Proceeding of the 6th international conference on hydrodynamics, Perth, November; 2004.

http://dx.doi.org/10.1201/b16815-20.

[12] API BULLETIN 2INT-MET, 2007, Interim Guidance on Hurricane Conditions in the Gulf of Mexico.

[13] Longuet-Higgins MS. 1980. On the distribution of the heights of sea waves: some effects of nonlinearity and finite band width. Journal of Geophysical Research: Oceans.85:1519-1523.

http://dx.doi.org/10.1029/JC085iC03p01519.

[14] Westerweel J. 1993. Digital particle image velocimetry: theory and application: TU Delft, Delft University of Technology. The Netherlands.

[15] Gui L, Merzkirch W. 2000. A comparative study of the MQD method and several correlation-based PIV evaluation algorithms. Experiments in Fluids.28:36-44. http://dx.doi.org/10.1007/s003480050005.

[16] Hirt CW, Nichols BD. 1981. Volume of fluid (VOF) method for the dynamics of free boundaries. Journal of computational physics.39:201-225. http://dx.doi.org/10.1016/0021-9991(81)90145-5.

[17] Choi J, Yoon SB. 2009. Numerical simulations using momentum source wave-maker applied to RANS equation model. Coastal Engineering.56:1043-1060. http://dx.doi.org/10.1016/j.coastaleng.2009.06.009.

[18] CD-Adapco. 2015. User Guide; STAR-CCM+10.06.

[19] Cooker M, Peregrine D. 1990. Violent water motion at breaking-wave impact. Proceedings of 22nd Conference on Coastal Engineering, Delft, 1990.

[20] Hull P, Müller G. 2002. An investigation of breaker heights, shapes and pressures. Ocean Engineering.29:59-79. h.ttp://dx.doi.org/10.1016/S0029-8018(00)00075-5

[21] Van der Meer J. 2002. Technical Report: Wave Runup and Wave Overtopping at Dikes. Technical Advisory Committee on Flood Defence. Delft. The Netherlands.

[22] Yasuda T, Mutsuda H, Mizutani N. 1997. Kinematics of overturning solitary waves and their relations to breaker types. Coastal Engineering.29:317-346

Submitted: $\quad$ 01.06.2016. Gang Nam Lee, Kwang Hyo Jung kjung@pusan.ac.kr, Young Jun Chae Department of Naval Architecture and Ocean Engineering,

Accepted: $\quad$ 08.08.2016. Pusan National University, Busan, Republic of Korea Il Ryong Park Department of Naval Architecture and Ocean Engineering, Dong-Eui University, Busan, Republic of Korea Sime Malenica, Bureau Veritas, Paris, France Yun Suk Chung, Bureau Veritas, Busan, Republic of Korea 